\title{
A BOUND FOR THE ERROR IN COMPUTING THE BESSEL FUNCTIONS OF THE FIRST KIND BY RECURRENCE
}

\author{
H. E. GOHEEN
}

It is sometimes useful to obtain the value of a Bessel function of the first kind of a fairly high integral order. Inasmuch as $J_{0}(x)$ and $J_{1}(x)$ are relatively easy to compute, even apart from the existence of numerous tables of $J_{0}(x)$ and $J_{1}(x)$ to considerable accuracy, one obvious method of attack is to use the recurrence relation,

$$
J_{n+1}(x)=\frac{2 n}{x} J_{n}(x)-J_{n-1}(x),
$$

the starting values being the already available values of $J_{0}(x)$ and $J_{1}(x)$. In using this formula, however, it is necessary to have a bound for the error in $J_{n}(x)$ caused by errors in $J_{0}(x)$ and $J_{1}(x)$. It is the purpose of this note to furnish such a bound.

Let $e_{n}(x)$ represent the difference between the value of $J_{n}(x)$ and the approximate value obtained by applying (1) to values which differ from $J_{0}(x)$ and $J_{1}(x)$ by errors $e_{0}(x)$ and $e_{1}(x)$, respectively. Since $e_{n}(x)$ is additive to the approximate value and since both the approximate and true values satisfy the recurrence formula, $e_{n}(x)$ must also satisfy the recurrence formula.

If exact values of $e_{0}(x)$ and $e_{1}(x)$ were known, an exact value of $e_{n}(x)$ could be obtained by the recurrence relation (1). However, by the nature of the problem bounds for the absolute values of $e_{0}(x)$ and $e_{1}(x)$, and in some cases their signs, are the only information available. These bounds cannot be used as starting values for recurrence to find a bound to the absolute value of $e_{n}(x)$ since in the relation (1) a substitution of the bounds on the right-hand side may yield a result whose absolute value is well within the bound for the absolute value of the left-hand side.

In the relation due to Lommel and Hankel, ${ }^{1}$

$$
2 / \pi x=Y_{n}(x) J_{n+1}(x)-Y_{n+1}(x) J_{n}(x),
$$

$Y_{n}(x)$ being the customary Bessel function of the second kind of order $n$, setting $n=0$, multiplying both sides by $(\pi x / 2) e_{0}(x)$, adding the null term $(\pi x / 2) e_{1}(x) Y_{0}(x) J_{0}(x)-(\pi x / 2) e_{1}(x) Y_{0}(x) J_{0}(x)$ and rearranging the terms give

Presented to the Society, November 2, 1946; received by the editors November 20, 1946, and, in revised form, March 22, 1947.

1 Watson, G. N., A treatise on the theory of Bessel functions, 2d ed., 1944, p. 77. 


$$
\begin{aligned}
e_{0}(x)= & (\pi x / 2) Y_{0}(x)\left[e_{0}(x) J_{1}(x)-e_{1}(x) J_{0}(x)\right] \\
& +(\pi x / 2) J_{0}(x)\left[e_{1}(x) Y_{0}(x)-e_{0}(x) Y_{1}(x)\right] .
\end{aligned}
$$

Setting $n=0$ in (2), multiplying by $(\pi x / 2) e_{1}(x)$, adding the null term $(\pi x / 2) e_{0}(x) Y_{1}(x) J_{1}(x)-(\pi x / 2) e_{0}(x) Y_{1}(x) J_{1}(x)$ to the right-hand side and rearranging give

$$
\begin{aligned}
e_{1}(x)= & (\pi x / 2) Y_{1}(x)\left[e_{0}(x) J_{1}(x)-e_{1}(x) J_{0}(x)\right] \\
& +(\pi x / 2) J_{1}(x)\left[e_{1}(x) Y_{0}(x)-e_{0}(x) Y_{1}(x)\right] .
\end{aligned}
$$

Since $e_{n}(x)$ satisfies the recurrence formula (1) as do $J_{n}(x)$ and $Y_{n}(x)$, it follows by complete induction that

$$
\begin{aligned}
e_{n}(x)= & (\pi x / 2) Y\left({ }_{n} x\right)\left[e_{0}(x) J_{1}(x)-e_{1}(x) J_{0}(x)\right] \\
& +(\pi x / 2) J_{n}(x)\left[e_{1}(x) Y_{0}(x)-e_{0}(x) Y_{1}(x)\right] .
\end{aligned}
$$

Since $Y_{n}(x)$ for fixed $x$ increases without any limit independent of $n$ as $n$ increases without limit, so does $e_{n}(x)$ unless the coefficient of $Y_{n}(x)$ vanishes. The values of $e_{0}(x)$ and $e_{1}(x)$ are the remainders in truncating series for $J_{0}(x)$ and $J_{1}(x)$, respectively, or the errors in obtaining these functions by asymptotic expansions, numerical quadratures or step-by-step integrations and therefore nothing is known of them except bounds for their absolute values and, in some cases, their signs. Hence, it can hardly be expected that the coefficient of $Y_{n}(x)$ vanishes; and almost always the error in $J_{n}(x)$ exceeds any bound independent of $n$ provided $n$ is large enough.

The function $(\pi x / 2) Y_{n}(x)$ is the sum of a negative function of $x$ with decreasing absolute value and a function whose absolute value is bounded by $\pi x / 2+1$, for ${ }^{2}$

$$
\begin{aligned}
\pi Y_{n}(x)= & -\sum_{m=0}^{<n / 2} \frac{(n-m-1) !}{m !}\left(\frac{x}{2}\right)^{-n+2 m} \\
& +\int_{0}^{\pi} \sin (x \sin \theta-n \theta) d \theta \\
& +2(-1)^{n+1} \int_{0}^{\infty} e^{-n t-x \sinh t} d t
\end{aligned}
$$

the first integral being less in absolute value than $\pi$ and the second less in absolute value than $2 \int_{0}^{\infty} e^{-n t-x t} d t$ or $2 /(n+x)$. Hence, $(\pi x / 2) J_{n}(x)\left[e_{1}(x) Y_{0}(x)-e_{0}(x) Y_{1}(x)\right]$ is in absolute value less than $(\pi x+3)\left|J_{n}(x)\right| \max \left\{\left|e_{0}\right|,\left|e_{1}\right|\right\}$, the bound being obtained by setting

2 Ibid. pp. 340-342. 
$n=0$ and $n=1$ in (6), determining bounds for $\left|Y_{0}(x)\right|$ and $\left|Y_{1}(x)\right|$, and using these bounds to obtain a bound for $\left|e_{1}(x) Y_{0}(x)-e_{0}(x) Y_{1}(x)\right|$.

The formula (6) is very difficult to use as a basis for estimation of $Y_{n}(x)$ if $n>10$. If tables of $Y_{0}(x)$ and $Y_{1}(x)$ are available, $Y_{n}(x)$ may be computed by use of the recurrence formula, or if tables of $Y_{n}(x)$ are available these may be used. It is unlikely that tables of $Y_{n}(x)$ will be available and not tables of $J_{n}(x)$ for $n>1$ and another procedure is necessary in this case. If only $J_{0}(x)$ and $J_{1}(x)$ are available, it is still possible by means of doubling the computation to obtain an auxiliary function $E_{n}(x)$, defined as the value obtained by recurring on 1 and -1 as starting values. Then from (5)

(7) $E_{n}(x)=(\pi x / 2) Y_{n}(x)\left[J_{1}(x)+J_{0}(x)\right]-(\pi x / 2) J_{n}(x)\left[Y_{0}(x)+Y_{1}(x)\right]$.

Then by use of the estimate

$$
(\pi x / 2)\left|J_{n}(x)\left[Y_{0}(x)+Y_{1}(x)\right]\right|<(\pi x+3)\left|J_{n}(x)\right|
$$

and by solving (7) for $Y_{n}(x)$, there results

$$
\frac{\pi x}{2} Y_{n}(x)=\frac{E_{n}(x)}{J_{1}(x)+J_{0}(x)}+\rho_{n}(x),
$$

with

$$
\left|\rho_{n}(x)\right|<\frac{(\pi x+3)\left|J_{n}(x)\right|}{\left|J_{1}(x)+J_{0}(x)\right|} .
$$

Hence, for all $x \geqq x_{0}>0$, denoting by $\phi(x)$ the polynomial in $1 / x$ which appears in the expansion of $(\pi x / 2) Y_{n}(x)$,

$$
\begin{aligned}
\left|e_{n}(x)\right| \leqq & (\pi x / 2)\left|Y_{n}(x)\right|\left|e_{1}(x) J_{0}(x)-e_{0}(x) J_{1}(x)\right| \\
& +(\pi x+3) \cdot\left|J_{n}(x)\right| \cdot \max \left(\left|e_{0}\right|,\left|e_{1}\right|\right) \\
\leqq & {\left[\left\{|\phi(x)|+\frac{\pi x}{2}+1\right\}\left\{\left|J_{0}(x)\right|+\left|J_{1}(x)\right|\right\}\right.} \\
& \left.+(\pi x+3) \cdot\left|J_{n}(x)\right|\right] \cdot \max \left(\left|e_{0}\right|,\left|e_{1}\right|\right) \\
\leqq & {\left[\left\{\left|\phi\left(x_{0}\right)\right|+\frac{\pi x}{2}+1\right\}\left\{\left|J_{0}(x)\right|+\left|J_{1}(x)\right|\right\}\right.} \\
& \left.+(\pi x+3)\left|J_{n}(x)\right|\right] \cdot \max \left(\left|e_{0}\right|,\left|e_{1}\right|\right) .
\end{aligned}
$$

However, 


$$
\frac{\pi x}{2} Y_{n}\left(x_{0}\right)=\phi\left(x_{0}\right)+r\left(x_{0}\right)
$$

or

$$
\left|\phi\left(x_{0}\right)\right| \leqq \frac{\pi x_{0}}{2}\left|Y_{n}\left(x_{0}\right)\right|+\frac{\pi x_{0}}{2}+1
$$

Finally,

$$
\left|\phi\left(x_{0}\right)\right| \leqq\left\{\frac{\left|E_{n}\left(x_{0}\right)\right|}{\left|J_{1}\left(x_{0}\right)+J_{0}\left(x_{0}\right)\right|}+\frac{\left(\pi x_{0}+3\right)\left|J_{n}\left(x_{0}\right)\right|}{\left|J_{1}\left(x_{0}\right)+J_{0}\left(x_{0}\right)\right|}+\frac{\pi x_{0}}{2}+1\right\}
$$

or

$$
\begin{array}{r}
\left|e_{n}(x)\right| \leqq\left[\left\{\frac{\left|E_{n}\left(x_{0}\right)\right|}{\left|J_{1}\left(x_{0}\right)+J_{0}\left(x_{0}\right)\right|}+\frac{\left(\pi x_{0}+3\right)\left|J_{n}\left(x_{0}\right)\right|}{\left|J_{1}\left(x_{0}\right)+J_{0}\left(x_{0}\right)\right|}\right.\right. \\
\left.+\frac{\pi\left(x_{0}+x\right)}{2}+2\right\}
\end{array}
$$

$$
\left.\cdot\left\{\left|J_{0}(x)\right|+\left|J_{1}(x)\right|\right\}+(\pi x+3) \cdot\left|J_{n}(x)\right|\right] \cdot \max \left(\left|e_{0}\right|,\left|e_{1}\right|\right)
$$

This bound will be almost exact for $e_{n}\left(x_{0}\right)$. To make it as good as possible, the value of $x_{0}$ should be taken as large as possible consistent with the values of $x$ for which $J_{n}(x)$ is to be tabulated. In case approximations to $J_{n}(x)$ and $J_{n-1}(x)$ are available for $n>1$, similar considerations will enable one to determine a bound to the error in $J_{k}(x)$ with $k>n$, obtained by recurrence on $J_{n}(x)$ and $J_{n-1}(x)$. 